\title{
Dual gene therapy with SERCA1 and Kir2.1 abbreviates excitation without suppressing contractility
}

\author{
Irene L. Ennis, ${ }^{1}$ Ronald A. Li, ${ }^{1}$ Anne M. Murphy, ${ }^{2}$ Eduardo Marbán, ${ }^{1}$ \\ and H. Bradley Nuss ${ }^{1}$ \\ ${ }^{1}$ Institute of Molecular Cardiobiology, and \\ ${ }^{2}$ Division of Pediatrics, Department of Medicine, The Johns Hopkins University School of Medicine, \\ Baltimore, Maryland, USA \\ Address correspondence to: H. Bradley Nuss, Institute of Molecular Cardiobiology, Ross 844, \\ The Johns Hopkins University, Baltimore, Maryland 21205, USA. \\ Phone: (410)614-0035; Fax: (410) 955-7953; E-mail: bradnuss@mail.jhmi.edu. \\ Irene L. Ennis's present address is: Centro de Investigaciones, Cardiovasculares, Facultad de Medicina, \\ Universidad Nacional de La Plata, La Plata, Argentina. \\ Received for publication May 24, 2001, and accepted in revised form December 27, 2001.
}

Heart failure is characterized by depressed contractility and delayed repolarization. The latter feature predisposes the failing heart to ventricular arrhythmias and represents a logical target for gene therapy. Unfortunately, unopposed correction of the delay in repolarization will decrease the time available for calcium cycling during each heartbeat, potentially aggravating the depression of contractility. Here we describe the development and application of a novel gene therapy strategy designed to abbreviate excitation without depressing contraction. The calcium ATPase SERCA1 was coexpressed with the potassium channel Kir2.1 in guinea pig hearts. Myocytes from the hearts had bigger calcium transients and shorter action potentials. In vivo, repolarization was abbreviated, but contractile function remained unimpaired. Dual gene therapy of the sort described here can be generalized to exploit opposing or synergistic therapeutic principles to achieve a tailored phenotype.

J. Clin. Invest. 109:393-400 (2002). DOI:10.1172/JCI200213359.

\section{Introduction}

Heart failure is a highly lethal syndrome, the two most common causes of mortality being sudden death and pump failure. Ventricular arrhythmias underlie many of the sudden deaths. Multiple lines of evidence implicate downregulation of $\mathrm{K}$ channel expression, with attendant delay of repolarization, in the pathogenesis of the ventricular arrhythmias associated with cardiomyopathy. K channel downregulation may initially be an adaptive response in the failing heart, given that prolongation of the action potential increases the time available for excitation-contraction coupling during each cardiac cycle. Gene therapy has been proposed as a therapeutic strategy to reverse $\mathrm{K}$ channel downregulation; nevertheless, an obvious risk of such therapy is a decline of the already compromised contractility of the failing heart. Our early monogenic attempt to normalize delayed repolarization in failing ventricular myocytes via overexpression of a foreign $\mathrm{K}$ channel proved the case in point (1). While overexpression of a noninactivating $\mathrm{K}$ channel effectively abbreviated repolarization, it adversely impacted excitation-contraction coupling by causing reduced cell shortenings (Figure 1) (1).

We thus sought to develop a strategy to reverse the $\mathrm{K}$ channel downregulation without depressing contractility. Bicistronic vectors enable the coexpression of two genes driven by a single promoter. We designed such a construct to coexpress the skeletal muscle calcium ATPase isoform SERCA1 with the K channel Kir2.1. Pre- vious studies have demonstrated the utility of SERCA gene delivery in boosting cardiac contractility by increasing calcium loading of the sarcoplasmic reticulum; here, we chose to use the SERCA1 isoform given its usual absence in the heart and its unique antigenicity (properties which facilitate the unambiguous documentation of transgene expression), as well as its high turnover rate relative to SERCA2 $(2,3)$. For the $\mathrm{K}$ channel, we selected Kir2.1, which encodes a cardiac inward rectifier channel. Kir2.1 is expected to abbreviate excitability by accelerating terminal repolarization (4). By itself, such an effect might prove deleterious for pump function, but we hypothesized that the addition of SERCA1 would offset the loss of contractility due to abbreviation of the action potential. The ideas were tested by expression of the dual gene therapy vector in the ventricle, measuring endpoints both in isolated cells (action potentials, membrane current, and calcium transients) and in vivo (electrocardiograms and echocardiograms).

\section{Methods}

Plasmid construction and adenovirus preparation. The adenovirus shuttle vectors pAdEGI, pAdEGI-Kir2.1, and pAdCGI-DBEcR have been described elsewhere $(5,6)$. The coding sequence from rat SERCA1a (kindly supplied by M. Periasamy, The Ohio State University, Columbus, Ohio, USA) was cloned into the first position of the adenovirus shuttle vector pAdEGI-Kir2.1 in place of the enhanced green fluorescence protein 


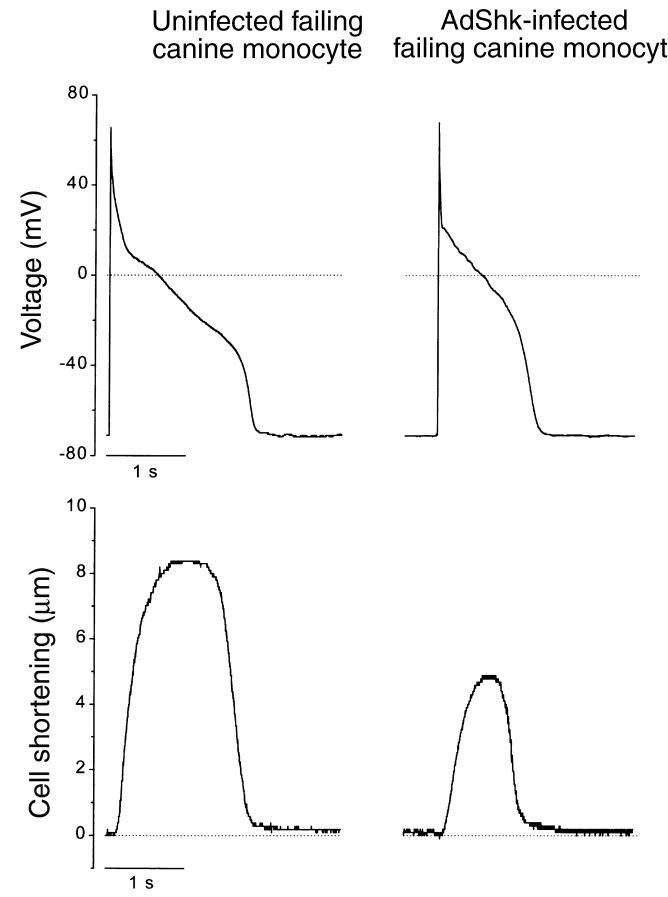

\section{Figure 1}

Adverse effects of monogenic K channel overexpression on contractility. Action potentials (upper panels) and contractions (lower panels) recorded $\left(22^{\circ} \mathrm{C}\right)$ in failing canine myocytes infected with AdShK and maintained in primary culture (1). Expression of ShK current shortened action potentials but also markedly blunted contractions (lower right panel). Prolonged action potentials and diminished $\mathrm{K}$ currents are characteristic of myocytes isolated from failing canine ventricles (18). This failing phenotype persists in primary culture (1).

(EGFP) sequence, to generate pAdSERCA1-Kir2.1. Adenovirus vectors were generated by Cre-lox recombination of purified $\psi 5$ viral DNA and shuttle vector DNA as described previously (5-7). The recombinant products were plaque-purified, expanded, and purified on $\mathrm{CsCl}$ gradients yielding concentrations on the order of $10^{10}$ plaque-forming units (PFU) per milliliter.

Animals. Adult guinea pigs (220-260 g) underwent direct intramyocardial adenovirus injection using a 30gauge needle as reported previously $(6,8,9)$. For patchclamp experiments, a single site within the anterior wall of the left ventricle was infiltrated under direct visualization three to five times with a total volume of $150 \mu \mathrm{l}$ of an adenovirus mixture containing $5 \times 10^{8} \mathrm{PFU}$ AdCGI-DBEcR and $5 \times 10^{8}$ PFU AdEGI (control group) or $5 \times 10^{8}$ PFU AdCGI-DBEcR and $5 \times 10^{8}$ PFU AdESERCA1-Kir2.1 (S-K group). For electrocardiogram (ECG) recordings and ultrasound studies, the left ventricle was injected in a more widespread manner at multiple sites (about ten sites, each site injected once) from the base to the apex of the anterior, lateral, and posterior wall, with a total volume of $220 \mu \mathrm{l}$ of the same adenovirus mixture, this time containing $8 \times 10^{8} \mathrm{PFU}$ AdCGI-DBEcR and $8 \times 10^{8} \mathrm{PFU}$ AdEGI (control group) or $8 \times 10^{8}$ PFU AdCGI-DBEcR and $8 \times 10^{8}$ PFU AdESERCA1-Kir2.1 (S-K group). After the chest was closed, animals were injected intraperitoneally with $40 \mathrm{mg}$ of the nonsteroidal ecdysone receptor agonist GS-E [N-(3methoxy-2-ethylbenzoyl)- $N^{\prime}$-(3,5-dimethylbenzoyl)- $N^{\prime}$ tert-butylhydrazine; kindly provided by Rohm and Haas Co., Spring House, Pennsylvania, USA], dissolved in 90 $\mu \mathrm{l}$ DMSO and $360 \mu \mathrm{l}$ sesame oil.

Myocyte isolation and electrophysiology. Seventy-two hours after myocardial injection, guinea pig left ventricular myocytes were isolated as described previously $(10,11)$. These enzymatic dissociations typically yielded $50-70 \%$ viable myocytes. The yield of infected myocytes, identifiable by their green fluorescence (see below), was about $2 \%$ as judged by visual assessments when cells were dispersed into the electrophysiologic recording chamber. The low yield of green viable myocytes was due to the limited efficiency of gene transduction by direct cardiac injection of adenovirus. Experiments were carried out using the whole-cell patch-clamp technique (12) with an Axopatch 200B amplifier (Axon Instruments Inc., Foster City, California, USA) while sampling at 10 $\mathrm{kHz}$ (for currents) or $2 \mathrm{kHz}$ (for voltage recordings) and filtering at $2 \mathrm{kHz}$. Pipettes had tip resistances of 2-4 $\mathrm{M} \Omega$ when filled with the internal recording solution. The inward rectifier $\mathrm{K}$ currents $\left(\mathrm{I}_{\mathrm{K} 1}\right)$ and calcium transients were recorded at room temperature $\left(\sim 21^{\circ} \mathrm{C}\right)$, while action potential (AP) recordings were obtained at $37^{\circ} \mathrm{C}$. APs were initiated by short depolarizing current pulses (2 ms, 200-600 pA) at $1 \mathrm{~Hz}$. AP duration (APD) was measured as the time from the overshoot to $50 \%$ or $90 \%$ repolarization $\left(\mathrm{APD}_{50}\right.$ or $\mathrm{APD}_{90}$, respectively). A xenon arc lamp was used to view green fluorescent protein (GFP) fluorescence at $488 / 530 \mathrm{~nm}$ (excitation/emission). Infected cells were recognized by their obvious green fluorescence. Although fluorescence was attributable to AdCGIDBEcR and not to AdESERCA1-Kir2.1 infection in the case of the $\mathrm{S}-\mathrm{K}$ group, it has been already shown by our laboratory that coinfection of the receptor and shuttle viruses is the rule rather than the exception (9). Given that we have shown that adenovirus infection itself does not modify the electrophysiology of guinea pig myocytes (9), patch-clamp experiments performed in uninfected (non-green) left ventricular myocytes isolated from AdESERCA1-Kir2.1-injected animals, as well as in green cells from AdEGI-injected hearts, were used as controls.

Cells were superfused with a physiological saline solution containing (in $\mathrm{mM}$ ): $138 \mathrm{NaCl}, 5 \mathrm{KCl}, 2 \mathrm{CaCl}_{2}$, 10 glucose, $0.5 \mathrm{MgCl}_{2}, 10$ HEPES; $\mathrm{pH}$ was adjusted to 7.4 with $\mathrm{NaOH}$. The micropipette electrode solution for $\mathrm{I}_{\mathrm{K} 1}$ recordings was composed of (in $\mathrm{mM}$ ): $140 \mathrm{KCl}$, $4 \mathrm{MgATP}, 5$ EGTA, $1 \mathrm{MgCl}_{2}$, 10 HEPES, pH adjusted to 7.4 with $\mathrm{KOH}$, while the one used for AP recordings contained (in $\mathrm{mM}$ ): $130 \mathrm{~K}$-glutamate, $10 \mathrm{KCl}, 5$ MgATP, $0.5 \mathrm{MgCl}_{2}, 10 \mathrm{NaCl}, 10$ HEPES, pH adjusted to 7.2 with $\mathrm{KOH}$. EGTA $(5 \mathrm{mM})$ was added to the pipette solution as indicated to record APs under highly Ca-buffered conditions. 


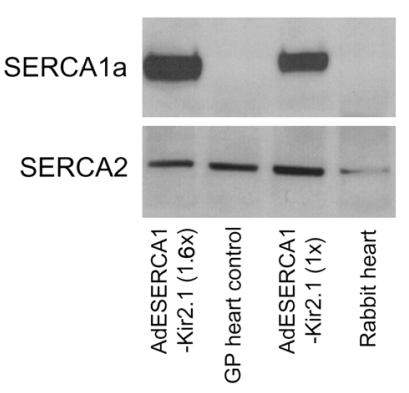

Figure 2

SERCA protein expression. When an mAb against SERCA1a (A52; ref. 14) was assayed, a specific band at about $100 \mathrm{kDa}$ was observed in samples from AdESERCA1-Kir2.1-injected hearts, while no signal was detected in control heart samples. Interestingly, the density of the band correlates well with the amount of virus injected $(1.6 \times \mathrm{vs}$. $1 \times)$. When a specific antibody against SERCA2 was assayed, the right band appeared in all heart samples (AdESERCA1-Kir2.1-injected and control) with the same magnitude. GP, guinea pig.

Calcium transients. The $\mathrm{K}^{+}$salt form of the $\mathrm{Ca}^{2+}$ indicator Indo-1 (Molecular Probes Inc., Eugene, Oregon, USA) was used to measure intracellular $\mathrm{Ca}^{2+}$ as previously described (13). Briefly, $\mathrm{Ca}^{2+}$ transients were elicited in isolated cardiomyocytes of the injected hearts in response to voltage steps (from -80 to 0 $\mathrm{mV})$ at a frequency of $1 \mathrm{~Hz}$. Indo- 1 fluorescence was excited at $365 \mathrm{~nm}$ with a xenon arc lamp and the emissions at $405 \mathrm{~nm}$ and $495 \mathrm{~nm}$ were collected with a two-channel photomultiplier tube (PMT) assembly (ESP Associates, Toronto, Canada). Fluorescence was digitized and stored simultaneously with the electrophysiological signals and analyzed using customwritten software (Ion View, written by B. O'Rourke). Indo-1 405/495-nm emission ratio was calculated after subtraction of cellular autofluorescence, which was recorded before rupturing the cell-attached patch. The internal pipette solution was composed of (in $\mathrm{mM}$ ): $130 \mathrm{~K}$-glutamate, $9 \mathrm{KCl}, 10 \mathrm{NaCl}, 0.5$ $\mathrm{MgCl}_{2}, 5 \mathrm{MgATP}, 10$ HEPES, 0.08 Indo-1, $\mathrm{pH}$ adjusted to 7.3 with $\mathrm{KOH}$.

Western blots. The ectopic expression of SERCA1a protein in hearts injected with AdESERCA1-Kir2.1, and the relative protein levels of SERCA2a and Kir2.1, were indexed by the immunoblot technique. For this purpose, crude homogenates of the left ventricle were prepared. Equal amounts of protein extract from the different groups of animals ( $20 \mu \mathrm{g} / \mathrm{lane})$ were subjected to electrophoresis and the separated proteins were transferred to nitrocellulose membranes (Novex immunoblot kit; Invitrogen Corporation, Carlsbad, California, USA). Blots were incubated with the appropriate $\mathrm{mAb}$ and in sequential steps with the corresponding peroxidase-conjugated secondary antibody (anti-mouse or anti-rabbit IgG; Amersham Biosciences Inc., Uppsala, Sweeden). The enhanced chemiluminescent detection system (Amersham) was used for signal detection. Relative protein level was determined by normalization to the level of actin, which was also used as an internal control. The following antibodies were used: A52 (14) (monoclonal SERCA1a antibody, kindly provided by $\mathrm{D}$. MacLennan, University of Toronto, Toronto, Ontario, Canada), MA3-910 (monoclonal SERCA2a antibody; Affinity BioReagents Inc., Golden, Colorado, USA), APC-026 (polyclonal anti-Kir2.1; Alomone Laboratories Ltd., Jerusalem, Israel), and AC-40 (monoclonal anti-actin; Sigma Chemical Co. St. Louis, Missouri, USA).

Immunoblots were also performed to evaluate how widespread SERCA1 expression was in animals that underwent multiple-site injections. Hearts were excised at 72 hours after viral injection and after ECG and echocardiography recordings were performed. Atria and all adjacent connective tissue were removed. The right ventricle was cut into two pieces and the left ventricle into six equal pieces. Homogenates of each sample were prepared and Western blots performed as described above using the monoclonal SERCA1a antibody A52 (14).

ECGs. Surface ECGs were recorded immediately after operation and 72 hours after intramyocardial injection as previously described $(9,15)$. Guinea pigs were sedated with methoxyflurane, and needle electrodes were placed under the skin. Electrode positions were optimized to obtain maximal amplitude recordings, enabling accurate measurements of QT intervals. ECGs were simultaneously recorded from standard lead II, and modified leads I and III. The positions of the needle electrodes were marked on the guinea pigs' skin after recording, to ensure exactly the same localization 72 hours later.

Cardiac echocardiography. Measurements were made 72 hours after intramyocardial injection. Guinea pigs were sedated with methoxyflurane and placed on a warming pad. Echocardiography images were obtained with a 6to $15-\mathrm{MHz}$ multifrequency probe using an Agilent Sonos 5500 ultrasound machine (Agilent Technologies Inc., Palo Alto, California, USA). Two-dimensional imaging-directed M-mode measurements of left ven-
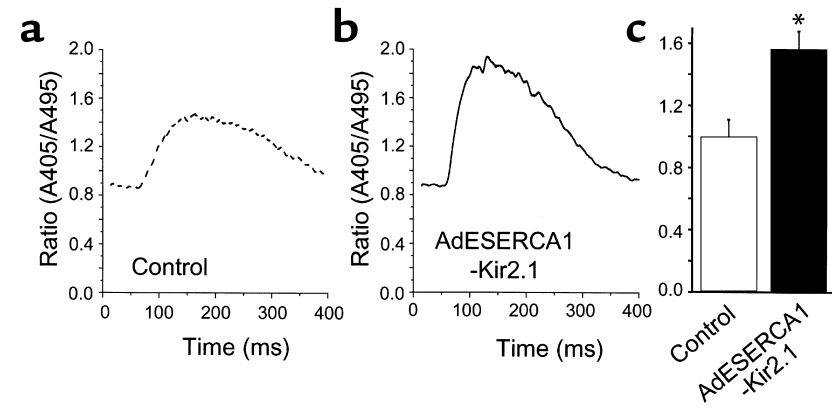

\section{Figure 3}

Intracellular $\mathrm{Ca}^{2+}$ transients. Representative $\mathrm{Ca}^{2+}$ transients elicited in control (a) and AdESERCA1-Kir2.1-infected myocytes (b) are shown. AdESERCA1-Kir2.1 infection increased the amplitude of the Indo-1 fluorescence ratio. (c) Average data are summarized ( $n=5$ for AdESERCA1-Kir2.1 group, and $n=8$ for control). ${ }^{*} P<0.05$, control versus $\mathrm{S}-\mathrm{K}$ group, $t$ test. 

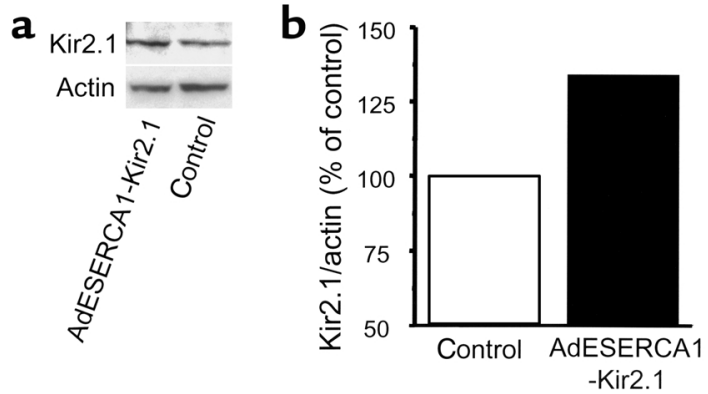

Figure 4

Kir2.1 protein expression. (a) Western blot analysis using a polyclonal antibody against Kir2.1 protein. Although the specific band at approximately $42 \mathrm{kDa}$ was present in both samples, its density was greater in the AdESERCA1-Kir2.1-injected guinea pig heart. (b) The relative expression of Kir2.1 normalized per actin protein for the same Western blot. Kir2.1 expression in the AdESERCA1-Kir2.1-injected heart was $30 \%$ higher than in control.

tricle end-diastolic (LVED) and left ventricle end-systolic (LVES) dimensions and septal and left ventricle free wall thickness were made. Heart rate was determined simultaneously with dimension measurements, and shortening fraction was calculated using the formula (LVED - LVES)/LVED.

Statistical analysis. Pooled data are presented as mean \pm SEM. Comparisons between groups were performed using $t$ test to compare unpaired data or two-way ANOVA to compare measurements made before and after ectopic expression in the same animals. $P$ values less than 0.05 were deemed significant.

\section{Results}

Ectopic expression of SERCA1 a protein in the infected hearts. We first sought to verify the expression of SERCA1 protein after intramyocardial injection in the S-K group. Western blot analysis was performed using cardiac homogenates to detect SERCA1a protein expression. Figure 2 shows that a SERCA1a-specific antibody (14) detected a band $(\sim 100 \mathrm{kDa})$ in the AdESERCA1Kir2.1-injected guinea pig hearts, but not in AdEGIinjected animals, where only SERCA2 was evident. Calcium transients. Cardiac myocytes isolated from humans and from several animal models of heart failure typically exhibit intracellular $\mathrm{Ca}^{2+}$ transients with decreased amplitudes $(13,16)$ consistent with decreased contractility, which is the hallmark of heart failure. The SERCA2a in the heart normally maintains appropriate $\mathrm{Ca}^{2+}$ loading of the sarcoplasmic reticulum (SR), thereby serving as a key modulator of releasable $\mathrm{Ca}^{2+}$ during excitation-contraction coupling. Heart failure is generally associated with decreased $\mathrm{Ca}^{2+}$ loading of the SR, so that supplementation of SERCA is a logical goal of therapy to reverse contractile dysfunction (17). Here, our primary goal was to preserve systolic function in the face of the abbreviation of excitation by coexpressed $\mathrm{K}$ channels.

To determine the functional consequences of SERCA1 ectopic expression in the absence of super- imposed changes of excitability, we measured $\mathrm{Ca}^{2+}$ transients during depolarizing voltage steps in freshly isolated cardiomyocytes from the left ventricle of injected guinea pigs. The results, shown in Figure 3, revealed a significant increase in the amplitude of Indo-1 fluorescence ratios in the AdESERCA1Kir2.1-infected cells compared with control myocytes (noninfected cells of the same hearts and AdEGIinfected myocytes) $(1.56 \pm 0.14, n=6$, vs. $1.00 \pm 0.12$, $n=8 ; P<0.05, t$ test). Thus, SERCA1 overexpression in the bicistronic vector enhanced the availability of activator $\mathrm{Ca}^{2+}$, a functional effect which may be salutary in heart failure and which would tend to offset the effects of $\mathrm{K}$ channel overexpression. Note that these calcium transients were deliberately measured under voltage clamp, with identical voltage step durations and amplitudes in both groups, to define changes of calcium availability independent of any changes in APD or morphology.

Overexpression of Kir2.1 protein. We next sought to verify the overexpression of Kir2.1 in the S-K group. Here, we cannot take advantage of differentially specific antibodies, as Kir 2.1 is normally expressed in the ventricle. Thus, we performed quantitative immunoblots using an antibody against Kir2.1 protein and compared the expression by densitometry to that of actin (which was assumed not to change). An increase of expression was detected in cardiac homogenates from AdESERCA1Kir2.1-infected hearts (Figure 4). Nevertheless, given the uncertainties of interpreting relatively small percent changes in immunoblots, we next sought functional evidence of an increased density of inward rectifier $\mathrm{K}$ channels in cardiomyocytes from the S-K group.

Overexpression of $I_{K 1}$ in guinea pig myocytes. As shown in Figure 5, 72 hours after injection and stimulation with GS-E, freshly isolated infected myocytes from the S-K group exhibit a significant increase in the inwardly rectifying $\mathrm{I}_{\mathrm{K} 1}$ density. The increase is particularly evident

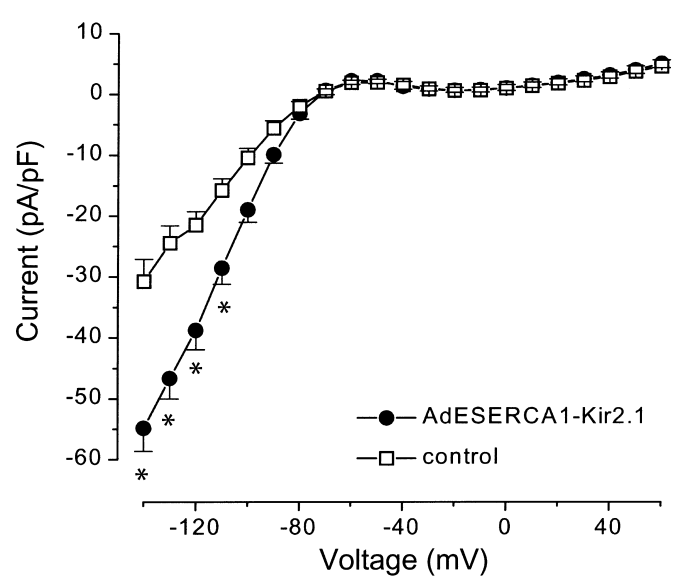

Figure 5

Inward rectifier $\mathrm{K}$ current $\left(\mathrm{I}_{\mathrm{K} 1}\right)$. AdESERCA1-Kir2.1-infected myocytes showed a significant increase in $I_{K 1}$ amplitude at negative membrane potentials when compared with control myocytes (AdEGI-infected or noninfected cells). ${ }^{*} P<0.01$, two-way ANOVA; $n \geq 8$ for each point. 

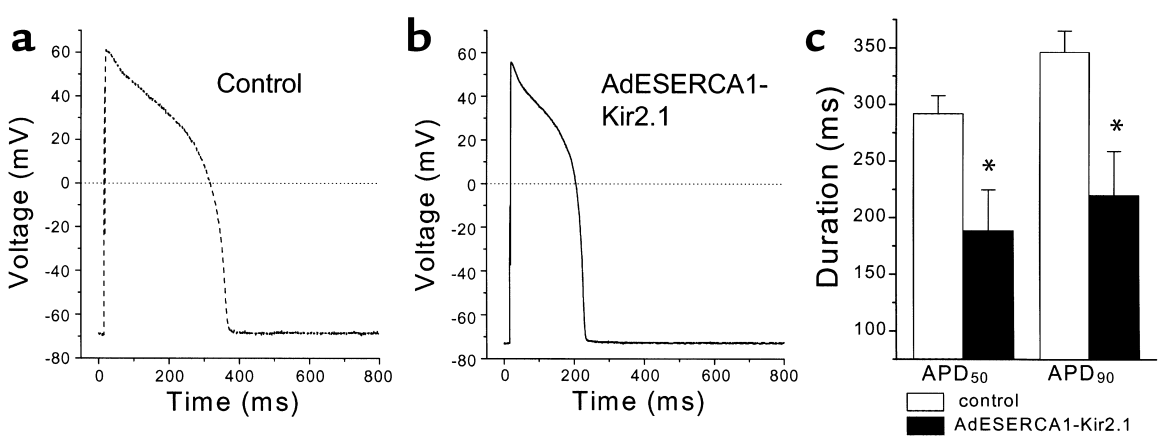

\section{Figure 6}

APD. ( $\mathbf{a}$ and $\mathbf{b}$ ) Representative action potentials elicited in control and AdESERCA1-Kir2.1-infected myocytes. AdESERCA1-Kir2.1 infection abbreviated the AP. (c) Average $\mathrm{APD}_{50}$ and $\mathrm{APD}_{90}$ data are summarized ( $n=8$ for AdESERCA1-Kir2.1 group, and $n=13$ for control). ${ }^{*} P<0.05$, AdESERCA1-Kir2.1 versus control, $t$ test.

at negative membrane potentials where conductance is large (i.e., $-54.8 \pm 3.8 \mathrm{pA} / \mathrm{pF}$ vs. $-30.7 \pm 3.6 \mathrm{pA} / \mathrm{pF}$ at $-140 \mathrm{mV}, n=8$ for each group; $P<0.001$, two-way ANOVA). We tested the functional importance of Kir2.1 overexpression at less negative potentials by examining the effect of AdESERCA1-Kir2.1 infection on $\mathrm{AP}$ repolarization.

$A P D$. Prolongation of the AP is characteristic of ventricular myocytes isolated from animals and humans with heart failure $(13,18-21)$. Changes in the APD result from alterations in the functional expression of depolarizing and repolarizing currents that are active during the plateau phase. Repolarization in the mammalian heart is achieved primarily by the activity of potassium-selective ionic currents. $\mathrm{I}_{\mathrm{K} 1}$ contributes to the terminal phase of repolarization, so an increase in the expression of these channels is a logical mechanism to shorten the APD. Figure 6 shows steady-state APs stimulated at $1 \mathrm{~Hz}$ in freshly isolated control and S-K ventricular myocytes at $37^{\circ} \mathrm{C}$ in the absence of added calcium buffer to the cytosol. The SERCAKir2.1-infected myocyte APs were significantly shorter than control myocyte APs, at both 50\% and $90 \%$ repolarization $\left(188.8 \pm 35.9 \mathrm{~ms}\right.$ vs. $292.2 \pm 15.6 \mathrm{~ms}\left[\mathrm{APD}_{50}\right]$ and $220.1 \pm 38.8 \mathrm{~ms}$ vs. $346.7 \pm 18.6 \mathrm{~ms}\left[\mathrm{APD}_{90}\right]$, for S-K myocytes $[n=8]$ vs. for control cells $[n=13]$, respectively; $P<0.05, t$ test).

We acknowledge that the electrophysiologic and $\mathrm{Ca}^{2+}$ handling pathways in the heart interact and, as such, may have impacted the degree of AP abbreviation achieved via genetic manipulation of SERCA1 and Kir 2.1 channels. We tested whether SERCA1 overexpression, by altering intracellular $\mathrm{Ca}^{2+}$ handling, had potentially increased or decreased the effect of Kir2.1 overexpression on abbreviation of APD via $\mathrm{Ca}^{2+}$ dependent modulation of membrane potential. Cellular AP recordings were made under identical conditions, with one exception: the addition of the cytosolic $\mathrm{Ca}^{2+}$ buffer EGTA $(5 \mathrm{mM})$. By buffering the $\mathrm{Ca}^{2+}$ transient, we eliminated $\mathrm{Ca}^{2+}$-dependent modulation of membrane potential exerted by the $\mathrm{Ca}^{2+}$-sensing actions of the L-type Ca current, the electrogenic
$\mathrm{Na}^{+} / \mathrm{Ca}^{2+}$ exchange, and other $\mathrm{Ca}^{2+}$ actions on currents. Under these $\mathrm{Ca}^{2+}$-buffered conditions, APs in control cells $\left(\mathrm{APD}_{50}=302.7 \pm 82.8 \mathrm{~ms}, \mathrm{APD}_{90}=376.8 \pm 79.8\right.$ $\mathrm{ms}, n=6)$ were slightly longer and APs in S-K cells $\left(\mathrm{APD}_{50}=147.9 \pm 28.9 \mathrm{~ms}, \mathrm{APD}_{90}=194.8 \pm 22.5 \mathrm{~ms}\right.$, $n=5)$ were shorter on average compared with APs recorded in the absence of any added $\mathrm{Ca}^{2+}$ buffer (not significant). Thus, via this experimental assessment, coexpression of SERCA1 moderated the impact of Kir2.1-overexpressed channels to shorten the AP.

These data demonstrate the intended therapeutic effect of overexpression of Kir2.1 at the cellular level. As long as SERCA-Kir2.1 overexpression does not aggravate contractile dysfunction, increased $I_{K 1}$ current would be expected to reduce the incidence of ventricular arrhythmias in failing hearts, by stabilizing repolarization and by suppressing triggered activity.

Widespread ectopic expression of SERCA1 a protein. Prior to performing cardiac function measurements, we ver-
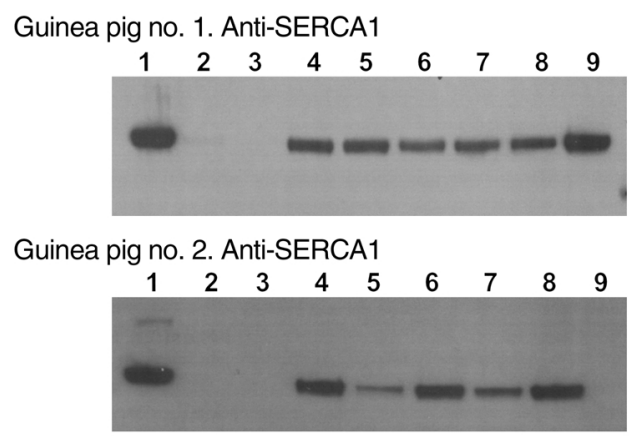

\section{Figure 7}

Widespread SERCA1 expression. Western blots from two different animals subjected to multiple-site injections of AdESERCA1-Kir2.1 throughout the left ventricle are shown. In both blots, the first lane corresponds to a homogenate of guinea pig skeletal muscle as a positive control for the SERCA1a-specific mAb A52 (14). Lanes 2 and 3 are the samples of the right ventricle, and lanes 4-9 correspond to each of six pieces into which the left ventricle was cut. Widespread expression of ectopic SERCA1a protein ( 100-kDa band) was obtained in the injected left ventricle, whereas no SERCA1a expression was observed in the noninjected right ventricle. 


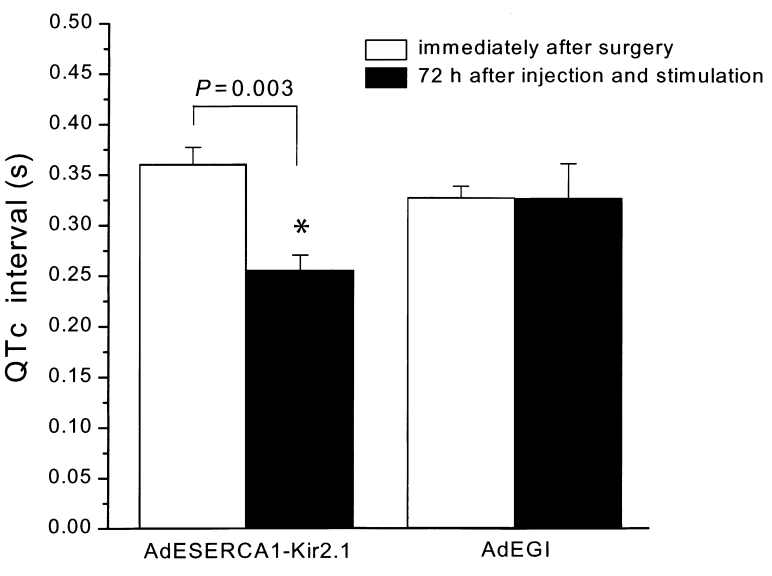

Figure 8

ECG analysis: QTc interval. A significant reduction was observed in the QTc interval in AdESERCA1-Kir2.1-injected animals (black bars). No changes occurred in the animals treated with AdEGI. ${ }^{*} P=0.003$, immediately after surgery versus 72 hours after injection and stimulation with GS-E, paired $t$ test.

ified widespread expression of SERCA1a protein throughout the left ventricle of animals that underwent multiple-site injections of AdESERCA1-Kir2.1. Western blot analysis was performed using homogenates of right and left ventricle and the SERCA1a-specific mAb A52 (14). The right ventricle was sectioned into two samples (Figure 7, lanes 2 and 3 ) and the left ventricle was cut into six similarly sized pieces (lanes 4-9). Figure 7 shows the Western blots from two different animals 72 hours after injection. In both blots, the first lane corresponds to a homogenate of guinea pig skeletal muscle used as a positive control for the antibody. Detection of the approximately $100-$ $\mathrm{kDa}$ SERCA1a-specific band in the immunoblots documents that widespread expression of ectopic SERCA1a protein was obtained throughout the entire left ventricle (lanes 4-9) in one animal and in five of six sections (lanes 4-8) of left ventricle in the other animal in which Western blot analysis was performed to assess the extent of protein expression. In contrast, no SERCA1a expression was observed in the noninjected right ventricle (lanes 2 and 3 ).

ECG recordings. Arrhythmias are a major cause of death in heart failure $(22,23)$. Cardiomyocytes from failing hearts reveal abnormalities in repolarization with prolongation of the QT interval, basically due to downregulation of $\mathrm{K}^{+}$currents, that favor the development of such arrhythmias. ECG recordings were performed in animals that had undergone widespread injection of the adenovirus vectors into the left ventricular myocardium. The QT interval was measured and corrected for heart rate using the following formula, with all measurements in sec-

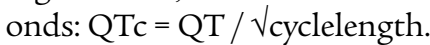

As shown in Figure 8, the QTc interval measured at 72 hours after injection and stimulation with GS-E was significantly abbreviated in the AdESERCA1-Kir2.1-injected guinea pigs compared with measurements made in the same animal immediately after surgery $(0.26 \pm 0.02 \mathrm{~s} v \mathrm{~s} .0 .36 \pm 0.02 \mathrm{~s}$; $P<0.03$, paired $t$ test). In contrast, no changes in the QTc interval were observed in the animals injected with AdEGI $(0.33 \pm 0.01 \mathrm{~s}$ vs. $0.33 \pm 0.03 \mathrm{~s}$; not significant). There was no difference, either, in the QTc interval recorded immediately after surgery between the two groups of animals (AdESERCA1-Kir2.1- and AdEGI-injected; data not shown).

Cardiac echocardiography. Table 1 displays the data from the cardiac echocardiography measurements. There were no significant differences in the left ventricular dimensions in the AdEGI versus the AdESERCA1-Kir2.1 animals. Left ventricular function as assessed by shortening fraction was also highly similar in the two groups. Heart rates were slightly higher, though not significantly so in the AdESERCA1-Kir2.1 animals. The septal and left ventricle free wall thicknesses were also not significantly different between the two groups (data not shown), indicating no pathologic hypertrophy developed in the animals. Although the AdESERCA1-Kir2.1 animals had significantly shortened APD and QT intervals, the function was not impaired, perhaps due to increased SR pumping efficiency related to the SERCA1 overexpression.

\section{Discussion}

To our knowledge, this is the first study to demonstrate the general utility of combination gene therapy for the heart, where both genes may be "therapeutic" and one is designed to offset the potentially adverse effects of the other. With the ultimate goal of normalizing the cardiac rhythm and increasing myocardial contractility in failing hearts without increasing vulnerability to arrhythmia, we created a dual gene therapy vector that coexpresses the skeletal muscle calcium ATPase isoform SERCA1 and the inward rectifier $\mathrm{K}$ channel Kir2.1. We hypothesized that overexpression of Kir2.1 alone would accelerate terminal repolarization but at the expense of contractility. Additional overexpression of SERCA1 would offset the loss of contractility due to abbreviation of the AP. In myocyte experiments, we have abbreviated APD and enhanced SR $\mathrm{Ca}^{2+}$ reuptake without creating unphysiologic or proarrhythmic effects. In vivo, we have abbreviated QT interval without impairing contractility. This research provides proof of principle that in vivo
Table 1

Echocardiography measurements in guinea pigs 72 hours after viral injection

\begin{tabular}{|c|c|c|c|c|c|c|}
\hline & & $\begin{array}{l}\text { LVED } \\
(\mathrm{cm})\end{array}$ & $\begin{array}{l}\text { LVES } \\
(\mathrm{cm})\end{array}$ & $\begin{array}{l}\mathrm{SF} \\
(\%)\end{array}$ & $\begin{array}{l}\text { Cycle length } \\
\text { (s) }\end{array}$ & $\begin{array}{l}\text { Heart rate } \\
\text { (beats } / \mathrm{min}\end{array}$ \\
\hline AdEGI & $\begin{array}{l}\text { Mean } \\
\text { SE }\end{array}$ & $\begin{array}{l}0.733 \\
0.033\end{array}$ & $\begin{array}{l}0.498 \\
0.038\end{array}$ & $\begin{array}{c}32.3 \\
2.8\end{array}$ & $\begin{array}{l}0.204 \\
0.008\end{array}$ & $\begin{array}{c}296 \\
13\end{array}$ \\
\hline $\begin{array}{l}\text { AdESERCA1 } \\
\text {-Kir2.1 }\end{array}$ & $\begin{array}{l}\text { Mean } \\
\text { SE }\end{array}$ & $\begin{array}{l}0.738 \\
0.043\end{array}$ & $\begin{array}{l}0.512 \\
0.036\end{array}$ & $\begin{array}{c}30.7 \\
2.1\end{array}$ & $\begin{array}{c}0.192 \\
0.0034\end{array}$ & $\begin{array}{c}310 \\
6\end{array}$ \\
\hline$t$ test $P$ value & & 0.937 & 0.806 & 0.67 & 0.244 & 0.366 \\
\hline
\end{tabular}


K channel gene transfer can modify cardiac repolarization without undermining contractility, a combination that may be useful as antiarrhythmic therapy.

The current analysis is limited to characterizing the acute effects of transgene expression. Whether endogenous gene expression is altered by acute overexpression of SERCA1 and Kir2.1 or by overexpression-induced alterations in myocyte function was not probed in this study. For example, we do not know if these types of genetic manipulations induce potential downstream stress or hypertrophic responses, which may become important considerations in the design of future chronic gene transduction studies.

Why not monogenic therapy? Solo gene therapy strategies, based upon selective overexpression of a single gene, may not suffice to treat multifactorial diseases such as heart failure. It is the interplay between the electrical and contractile components of heart failure that confounds the design of successful monogenic treatments. Our early results with novel antiarrhythmic monogenic gene therapy, based upon manipulation of a select $\mathrm{K}$ channel gene and targeted to correct the electrical abnormalities alone, adversely impacted contractility (1), which is already depressed in heart failure. In our earliest study, we expressed a noninactivating Shaker potassium channel (ShK) in ventricular myocytes isolated from cardiomyopathic adult canine hearts (1). A low level of ShK expression reversed the abnormal phenotype of the failing myocyte by shortening APD sufficiently to modify the failing AP waveform to resemble closely that of the normal myocytes (1). Moderate and higher levels of ShK expression abbreviated the failing AP markedly and reduced the size of contractions in AdShK-infected cells (Figure 1). In general, shortenings in AdShK-infected cells became smaller as the level of expressed ShK current increased and were roughly half as large as in control cells (1). At the highest levels of ShK expression achieved, APs were so severely modified that excitation was effectively uncoupled from contraction. In the present study, we have shown that a $\mathrm{K}$ channel gene and an $\mathrm{SR} \mathrm{Ca}^{2+}$ ATPase gene can be overexpressed in tandem to offset the contractile depression associated with monogenic $\mathrm{K}$ channel overexpression.

Rationalizing global effects on repolarization. One paradox in the current work is the ability to observe global correlates of transduction (QT interval abbreviation), while the proportion of GFP-positive isolated myocytes is relatively low $(2-3 \%$ based upon visual assessments of myocytes isolated from single-site-injected hearts). Several considerations help to resolve this paradox. First, multiple sites $(\sim 10)$ in the heart were injected (once per site) in the experiments designed to assess ECGs and echocardiograms whereas only a single site was infiltrated (albeit several times) when the sole objective was the isolation of myocytes for electrophysiological study. Second, the proportion of transduced myocytes may have been underestimated by our insistence on selecting only those cells that exhibited bright green fluorescence, which would have excluded cells that had taken up and expressed lower levels of the viral constructs. Third, it should be recognized that the heart is an electrical syncytium, and that the electrical effects of transduction will be felt as far away as approximately $1 \mathrm{~mm}$ or more from any given injected cell due to electrotonic effects through low-resistance gap junctions $(24,25)$ Thus, relatively low, conservative estimates of transduction efficiency after myocyte isolation are not necessarily incompatible with modification of global repolarization in vivo.

Our successful modification of cardiac excitability and contractility of the normal heart in vivo using dual gene therapy will motivate our future efforts to offset electrical and contractile abnormalities of common acquired forms of heart disease and to prevent fatal arrhythmias. This type of dual gene therapy can be generalized to exploit opposing or synergistic therapeutic principles to achieve a tailored phenotype.

\section{Acknowledgments}

We thank Michelle K. Leppo for her expert technical assistance. Research for this study was supported by the following sources: a fellowship award from Universidad Nacional de La Plata Argentina to I.L. Ennis; a personnel award from the Heart and Stroke Foundation of Canada to R.A. Li; NIH grant R01 HL-63038 to A.M. Murphy; NIH grant P50 HL-52307 to E. Marbán; and American Heart Association Scientist Development Grant 9730261N and NIH grant 1R01 HL66381-01 to H.B. Nuss. E. Marbán holds the Michel Mirowski M.D. Professorship of Cardiology at The Johns Hopkins University.

1. Nuss, H.B., et al. 1996. Reversal of potassium channel deficiency in cells from failing hearts by adenoviral gene transfer: a prototype for gene therapy for disorders of cardiac excitability and contractility. Gene Ther. 3:900-912.

2. Cavagna, M., O’Donnell, J.M., Sumbilla, C., Inesi, G., and Klein, M.G. 2000. Exogenous Ca2+-ATPase isoform effects on Ca2+ transients of embryonic chicken and neonatal rat cardiac myocytes. J. Physiol. 528:53-63.

3. Sumbilla, C., et al. 1999. Comparison of SERCA1 and SERCA2a expressed in COS-1 cells and cardiac myocytes. Am. J. Physiol. 277:H2381-H2391.

4. Nichols, C.G., Makhina, E.N., Pearson, W.L., Sha, Q., and Lopatin, A.N. 1996. Inward rectification and implications for cardiac excitability. Circ. Res. 78:1-7.

5. Johns, D.C., Marx, R., Mains, R.E., O’Rourke, B., and Marban, E. 1999. Inducible genetic suppression of neuronal excitability. J. Neurosci. 19:1691-1697.

6. Hoppe, U.C., Marban, E., and Johns, D.C. 2000. Adenovirus-mediated inducible gene expression in vivo by a hybrid ecdysone receptor. Mol. Ther. 1:159-164.

7. Hardy, S., Kitamura, M., Harris-Stansil, T., Dai, Y., and Phipps, M.L. 1997. Construction of adenovirus vectors through Cre-lox recombination. J. Virol. 71:1842-1849.

8. Quinones, M.J., et al. 1996. Avoidance of immune response prolongs expression of genes delivered to the adult rat myocardium by replicationdefective adenovirus. Circulation. 94:1394-1401.

9. Hoppe, U.C., Marban, E., and Johns, D.C. 2000. Molecular dissection of cardiac repolarization by in vivo Kv4.3 gene transfer. J. Clin. Invest. 105:1077-1084.

10. Hoppe, U.C., Johns, D.C., Marban, E., and O'Rourke, B. 1999. Manipulation of cellular excitability by cell fusion: effects of rapid introduction of transient outward $\mathrm{K}+$ current on the guinea pig action potential. Circ. Res. 84:964-972.

11. Mitra, R., and Morad, M. 1986. Two types of calcium channels in guinea pig ventricular myocytes. Proc. Natl. Acad. Sci. USA. 83:5340-5344. 
12. Hamill, O.P., Marty, A., Neher, E., Sakmann, B., and Sigworth, F.J. 1981. Improved patch-clamp techniques for high-resolution current recording from cells and cell-free membrane patches. Pflugers Arch. 391:85-100.

13. O'Rourke, B., et al. 1999. Mechanisms of altered excitation-contraction coupling in canine tachycardia-induced heart failure. I. Experimental studies. Circ. Res. 84:562-570.

14. Zubrzycka-Gaarn, E., MacDonald, G., Phillips, L., Jorgensen, A.O., and MacLennan, D.H. 1984. Monoclonal antibodies to the $\mathrm{Ca} 2++\mathrm{Mg} 2+-$ dependent ATPase of sarcoplasmic reticulum identify polymorphic forms of the enzyme and indicate the presence in the enzyme of a classical high-affinity Ca2+ binding site. J. Bioenerg. Biomembr. 16:441-464.

15. Hoppe, U.C., Marban, E., and Johns, D.C. 2001. Distinct gene-specific mechanisms of arrhythmia revealed by cardiac gene transfer of two long QT disease genes, HERG and KCNE1. Proc. Natl. Acad. Sci. USA 98:5335-5340.

16. Beuckelmann, D.J., and Erdmann, E. 1992. Ca(2+)-currents and intracellular $[\mathrm{Ca} 2+]$ i-transients in single ventricular myocytes isolated from terminally failing human myocardium. Basic Res. Cardiol. 87(Suppl. 1):235-243.

17. Hajjar, R.J., del Monte, F., Matsui, T., and Rosenzweig, A. 2000. Prospects for gene therapy for heart failure. Circ. Res. 86:616-621.

18. Kaab, S., et al. 1996. Ionic mechanism of action potential prolongation in ventricular myocytes from dogs with pacing-induced heart failure. Circ. Res. 78:262-273.

19. Beuckelmann, D.J., Nabauer, M., and Erdmann, E. 1992. Intracellular calcium handling in isolated ventricular myocytes from patients with terminal heart failure. Circulation. 85:1046-1055.

20. Li, H.G., Jones, D.L., Yee, R., and Klein, G.J. 1992. Electrophysiologic substrate associated with pacing-induced heart failure in dogs: potential value of programmed stimulation in predicting sudden death. J. Am. Coll. Cardiol. 19:444-449.

21. Vermeulen, J.T., et al. 1994. Triggered activity and automaticity in ventricular trabeculae of failing human and rabbit hearts. Cardiovasc. Res. 28:1547-1554

22. Luu, M., Stevenson, W.G., Stevenson, L.W., Baron, K., and Walden, J. 1989. Diverse mechanisms of unexpected cardiac arrest in advanced heart failure. Circulation. 80:1675-1680.

23. Stevenson, W.G., Stevenson, L.W., Middlekauff, H.R., and Saxon, L.A. 1993. Sudden death prevention in patients with advanced ventricular dysfunction. Circulation. 88:2953-2961.

24. Noble, D. 1975. The initiation of the heartbeat. Clarendon Press. Oxford, United Kingdom. 1-38.

25. Weidman, S. et al. 1970. Electrical constants of trabecular muscle from mammalian heart. J. Physiol. 210:1041-1054. 